\title{
SUPER-RESOLUTION IMAGES ON MOBILE SMARTPHONE AIMED AT 3D MODELING
}

\author{
L. Inzerillo ${ }^{1}$ \\ ${ }^{1}$ University of Palermo - Department of Engineering, Italy - laura.inzerillo@unipa.it
}

Commission II

KEY WORDS: smart phone survey, super resolution of images, photogrammetry, algorithms.

\begin{abstract}
:
In these last years have been calculated several general algorithms to process the images acquired by mobile devices. Nevertheless, they don't always work at their full effectiveness due the numerous constraints and external issues. The latest generation smartphones, pushed by higher user expectations, have increasingly performing camera functions, in particular way, about the always increasing resolution, with a large number of pixels to process or two cameras for stereo view. Considering that pixels in an image sensor synthetizes the number of incoming photons, taking a photograph with a digital camera means to applying a low-pass filter to a scene where the tiny textures, such as characters that measure a few pixels, are observed blurry. This happens when using digital zoom or when the visibility is compromised or during the night artificial lights. For all these reasons the conventional image converter resolution algorithms (as bilinear interpolation algorithm) don't work with the high-frequency information of a scene once lost. All these aspects are more relevant if we are taking photos to carry out a 3D scenario. Indeed, the 3D model will have an higher geometric accuracy if the image resolution will be higher. Super-Resolution algorithms (SRa) are classified into two categories: (1) approaches that reconstruct a high-resolution image from itself and (2) approaches that register multiple low-resolution images to interpolate sub-pixel information. In this paper we verify the geometric accuracy of a 3D model, when using the Morpho Super-Resolution ${ }^{\mathrm{TM}}$ algorithm, also in critical condition. This algorithm doesn't require pixel shift, indeed, some cameras have a functionality named pixel shift, which captures multiple images while shifting the image sensor.
\end{abstract}

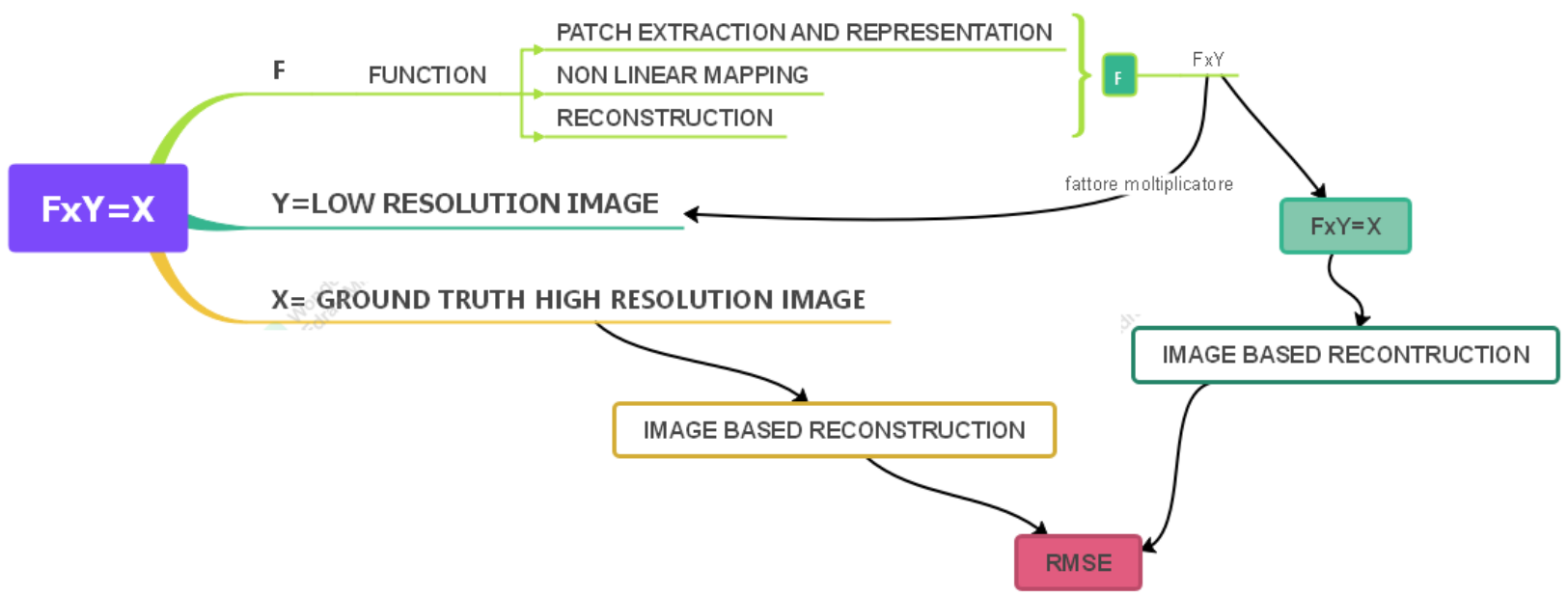

Figure 1. Flow Chart Methodology.

\section{INTRODUCTION}

The super-resolution (SR) of the images, that allows to carry out a high-resolution image from a single low resolution image, presents many solutions that can be associated to any given lowresolution pixel. It is an inverse problem that, nowadays, is not determined since it presents several solutions. To overcome this problem, it is necessary to provide deep preliminary information in order to constrain the space of the solution. In 2015, Chao et al. (Chao et al., 2015), proposed a convolutional neural network that named Super- Resolution Convolutional Neural Network (SRCNN) and demonstrated that it was more performer than the bicubic and the sparse-coding-based (SC) (Figure 2).

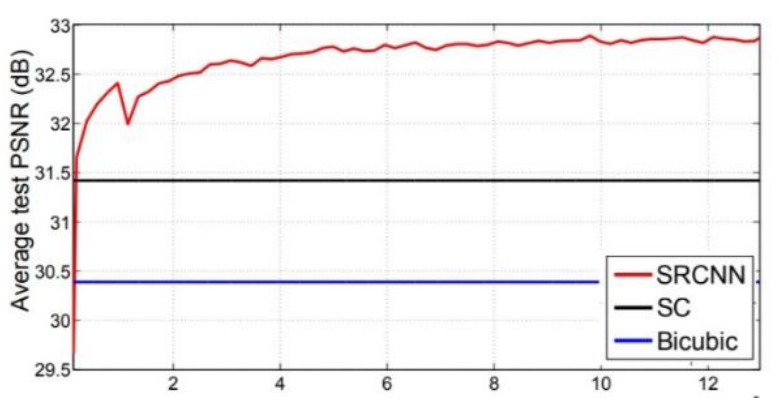

Figure 2. The Super-Resolution Convolutional Neural Network (SRCNN) surpasses the bicubic baseline with just a few training iterations, and outperforms the sparse-coding-based method (SC) with moderate training. 
The problem of the Super Resolution image is typical of the computer vision field. Nevertheless, frequently, during the photogrammetric survey in particular light or geometric conditions or big distance from the object, we carry out images that give problems during the generation of the mesh of the $3 \mathrm{D}$ model and the RMSE final value is not acceptable (Inzerillo, 2020). Another typical sample where the images are at a low resolution is when we need to have the $3 \mathrm{D}$ model by ancient photos of a no longer existing building. And once again, with UAV images for road maintenance (Roberts et al., 2020). The goal is to demonstrate that the geometric accuracy, of the 3D model carried out from photogrammetric survey with super resolution images, has a RMSE value close to the truth model than the one carried out without any correction (Fan et al., 2019). For this reason I chosen the façade of a building in the historical centre of the city. The typical difficulties linked to a façade are the accessibility to make the dataset and the upper side. When the surface to reconstruct is approximately flat, as in this case, the transformation between image and object coordinates is provided by a planar homography (Wu, 2013). This step is described by a $3 \times 3$ non-singular matrix $\mathrm{H}$ :

$$
\left.\mathrm{x}=\left[\begin{array}{l}
x \\
y \\
1
\end{array}\right]=\left[\begin{array}{lll}
h_{1} & h_{2} & h_{3} \\
h_{4} & h_{5} & h_{6} \\
h_{7} & h_{8} & h_{9}
\end{array}\right] \| \begin{array}{l}
X \\
Y \\
1
\end{array}\right]=\mathbf{H X}
$$

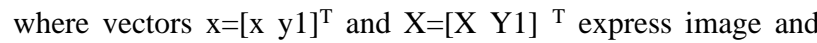
object points in homogenous coordinates, respectively. In this case, the GRS has only two degree-of-freedom (DoF), being the object planar. Eq. (1) shows two fundamental aspects: the first one is that just one image allows us to carry out the reconstruction of an object that is planar and the second one is that, since $\mathrm{H}$ is non-singular, the inverse transformation is always processed. On the other hand, object coordinates (X) are not always available. In this case, homography can be estimated as proposed in Barazzetti (2011).

By measuring a real distance will be scaled the 3D model and, apart this measure (Fan et al., 2019), the information to reconstruct $3 \mathrm{D}$ coordinates are enough without acquiring metric data (e.g. known ratios of distances and angles, object points) (Remondino et al., 2017; Rublee et al., 2011).

\section{RELATED WORKS}

The SRa topic is mainly developed in Computer Vision field. For this reason, the references reported below are in $\mathrm{CV}$ journal. The SRa have been verified and evaluated in Yang et al (Yang et al., 2014). Among them, the example-based methods (Glasner et al., 2009; Kim et al., 2010; Timofte et al., 2013) achieved the stateof-the-art performance. Other researchers developed some algorithms to accelerate the implementation (Freedman et al., 2011; Yang et al., 2014). Other mapping functions such as kernel regression (Kim et al., 2010), simple 3 function (Yang et al., 2013), random forest (Timofte et al., 2014) and anchored neighbourhood regression are proposed to further improve the mapping accuracy and speed. The sparse coding-based method and its several improvements (Timofte et al., 2014; Yang et al., 2014; Yang et al., 2013) are among the state-of-the-art SR methods nowadays. In these methods, the patches are the focus of the optimization; the patch extraction and aggregation steps are considered as pre/post-processing and handled separately.

\section{METHODOLOGY}

\subsection{Super resolution image processing}

Single image super-resolution (SISR) aims to reconstruct a highresolution image ISR from a single low-resolution image ILR. Generally, the relationship between ILR and the original highresolution image IHR can vary depending on the situation (Jin et al., 2020). Many studies assume that ILR is a bicubic down sampled version of IHR, but other degrading factors such as blur, decimation, or noise can also be considered for practical applications (Lowe, 2004).

To obtain a ISR needs to increase the spatial resolution of images or simply increasing the number of pixel rows/columns or both in the image. Several methods have been developed: the interpolation-based methods - Image interpolation (image scaling), that refers to resizing digital images and is widely used by image-related applications (Yi et al., 2016). The traditional methods include nearest-neighbor interpolation, linear, bilinear, bicubic interpolation, etc.; Nearest-neighbor Interpolation - The nearest-neighbor interpolation that is a simple and intuitive algorithm that selects the value of the nearest pixel for each position to be interpolated regardless of any other pixels; Bilinear Interpolation - The bilinear interpolation (BLI) that shows much better performance than nearest-neighbor interpolation while keeping a relatively fast speed and last but not least the Bicubic Interpolation - Similarly, the bicubic interpolation (BCI) that performs cubic interpolation on each of the two axes compared to BLI, the BCI takes $4 \times 4$ pixels into account, and results in smoother results with fewer artifacts but much lower speed.

Starting from the high resolution image, the low resolution image is modelled using the formula (2) where $\mathrm{X}$ is the high resolution image, $\mathrm{Y}$ is the low resolution image, $\mathrm{F}$ is the degradation function and $\sigma$ is the noise.

$$
\mathrm{X}=\mathrm{F}(\mathrm{Y} ; \sigma)
$$

The degradation parameters $\mathrm{D}$ and $\sigma$ are unknown; only the high resolution image and the corresponding low resolution image are provided. In order to find the inverse function of degradation, the precise task was given to the neural network, just using the HR and LR image data (Verdie et al., 2015).

Learning the end-to-end mapping function $\mathrm{F}$ requires the estimation of network parameters $\Theta=\{\mathrm{W} 1, \mathrm{~W} 2, \mathrm{~W} 3, \mathrm{~B} 1, \mathrm{~B} 2$, $\mathrm{B} 3\}$. In this way was achieved the aim of minimizing the MSE between the reconstructed images $\mathrm{F}(\mathrm{Y} ; \Theta)$ and the high resolution images $\mathrm{X}$, used at the start. The algorithm developed (formula 3) uses Mean Squared Error as the loss function, starting from a series of high resolution images $\{\mathrm{Xi}\}$ and the corresponding low resolution ones:

$$
\mathrm{L}(\Theta)=1 \text { n } \mathrm{X}_{\mathrm{ni}}=1\left\|\mathrm{~F}\left(\mathrm{Y}_{\mathrm{i}} ; \Theta\right)-\mathrm{X}_{\mathrm{i}}\right\| 2
$$

where $\mathrm{n}$ is the number of training samples. The strategy adopted, to use MSE as the loss function, allow us to achieved a high PSNR that is a widely-used metric for quantitatively evaluating image restoration quality, and it is related to the perceptual quality in partial quantity. Nevertheless, Chao Dong et al., (Chao Dong et al,. 2015) demonstrated that when they used the formula (4) where are calculated the weight matrices, observed satisfactory performance in fact, the loss is minimized using stochastic gradient descent with the standard backpropagation.

$$
\Delta_{\mathrm{i}+1}=0.9 \cdot \Delta_{\mathrm{i}}-\eta \cdot \frac{\partial \mathrm{L}}{\partial W_{i}^{l}}, W_{i}^{l}=W_{i}^{l}+\Delta_{\mathrm{i}+1}
$$


where $1 \in\{1,2,3\}$ and $i$ are the indices of layers and iterations, $\eta$ is the learning rate, and $\frac{\partial \mathrm{L}}{\partial W_{i}^{l}}$ is the derivative. The filter weights of each layer are initialized by drawing randomly from a Gaussian distribution with zero mean and standard deviation 0.001 (and 0 for biases). The learning rate is $10-4$ for the first two layers, and $10-5$ for the last layer. They empirically found that a smaller learning rate in the last layer is important for the network to converge.

Using the interpolation offered by the algorithm it was possible increase the quality of the images and the corresponding 3D model.

In this paper, the developed algorithm has been used to demonstrate if the relative quality of the carried out 3D model of architectural artefact responds to the improvement of the images quality and how much is this improvement. The façade of an ancient building in the historical centre of Palermo (Sicily, Italy) was chosen (Figure 3) (Shen et al., 2018)). The data sets are two: the first one with low resolution images and the other one with high resolution image. Then the low resolution data sets has been modified using the Morpho Super-Resolution algorithm and compared with high one as ground truth source.

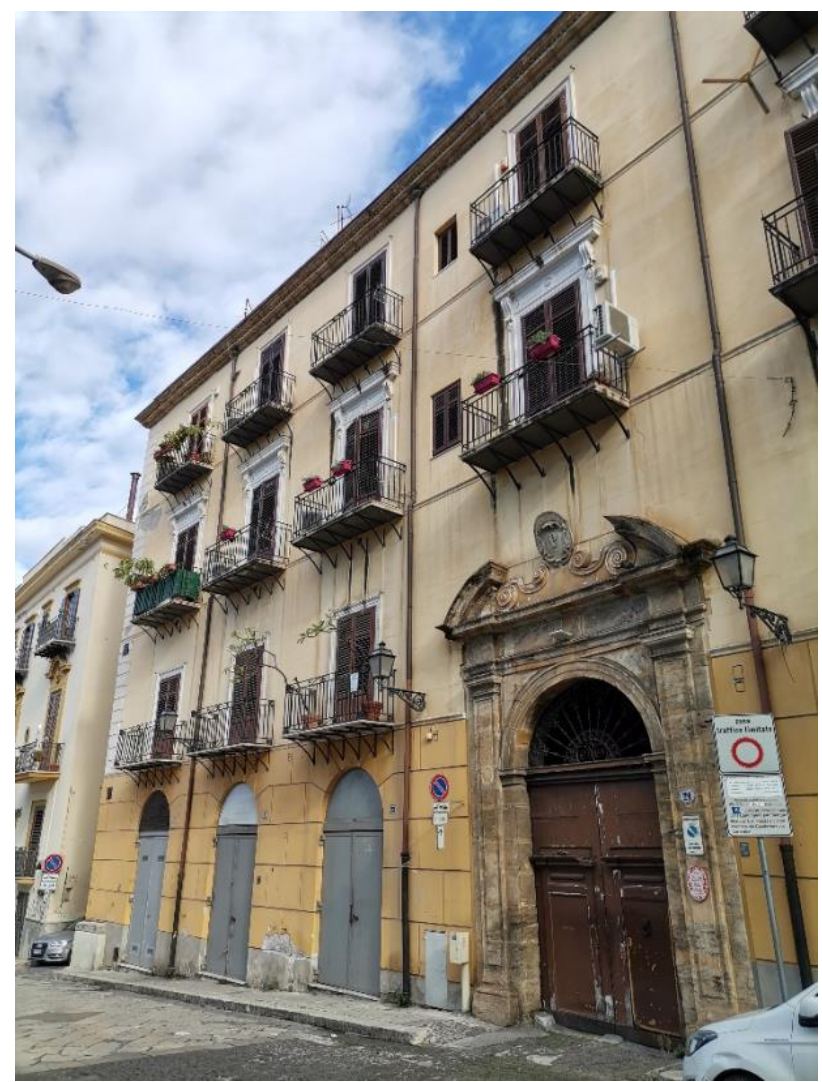

Figure 3: Low Image resolution of the façade of an ancient building.

The Morpho Super-Resolution ${ }^{\mathrm{TM}}$ algorithm is supported by a software, named Morpho Super-Resolution ${ }^{\mathrm{TM}}$ that includes the following functions:

- Video stabilization Movie Solid: MovieSolid cancels out camera shake electronically by cropping an area of an image. This technique is called electronic image stabilization. There is also a technique called optical image stabilization which mechanically moves the lens to compensate for camera shake (Luo et al., 2020). The biggest advantage of electronic image stabilization over optical stabilization is that it requires no special hardware, so it can be installed in inexpensive products. The disadvantage is that the image must always be cropped, which narrows the effective angle of view.

- Image Stabilization PhotoSolid: The value in PhotoSolid is that it provides sharp images without camera shake or noise (Schönberger et al. 2017). Those who have a single-lens reflex camera may know well that camera shake and noise are the counterparts related with image degradation. Cameras, not limited to those of mobile phones, are devices that measure the amount of incident light. In other words, the more light enters a camera, the brighter the image is, and vice versa. When taking photos in a dark scene such as a night scene, noise turns up more than incoming light, which results in noisy images.

- Image Enhancement by AI Based Segmentation and Pixel Filtering "Morpho Semantic FilteringTM: "Morpho Semantic Filtering" is an image enhancement software that uses AI based segmentation and pixel filtering (Moulon et al., 2016). In 2020, it won the Best AI Software/Algorithm Award at a Vision Product of the Year Awards 2020 sponsored by the Edge AI's international-industry organization.

- Fast AI Inference Engine "SoftNeuro®" "SoftNeuro®" operates in multiple environments, utilizing learning results that have been obtained through a variety of deep learning frameworks. It's easy to use, it's fast and it can be used anywhere. Using this framework doesn't require you to know anything about Deep Learning. Another advantage of SoftNeuro is that it can import from various frameworks and run fast on various architectures. Also, SoftNeuro is both flexible and fast because of the separation of the layer and its execution pattern, which is a concept of routine.

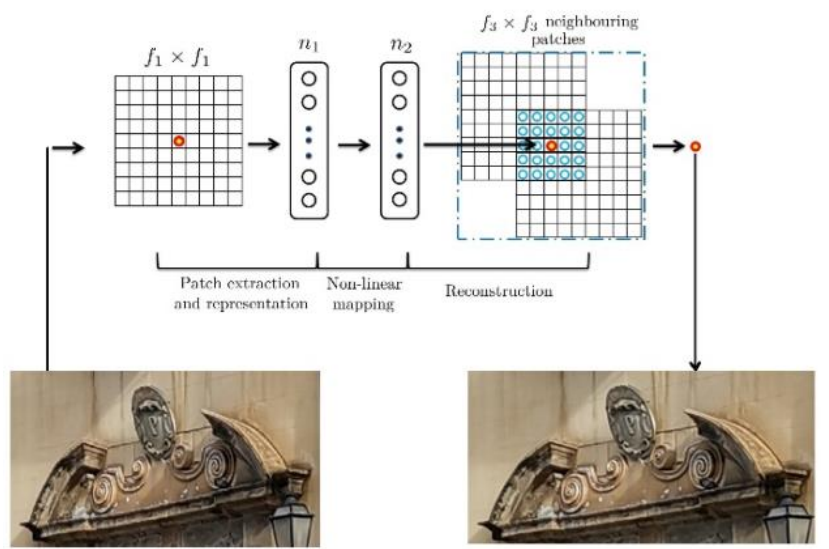

Figure 4: In the picture, there are two different layers: $f_{l} x f_{l}$ and $f_{3} x f_{3}$. Starting from a low -resolution image (Y), the first functional layer of the process extracts a set of feature maps. The last layer combines the predictions within a spatial neighbourhood to produce the final high-resolution image $F(Y)$ called Super-Resolution Image. Between the two different layers, there is the non- linear mapping. This method is the sparsecoding-based one in the view of a convolutional neural network. 


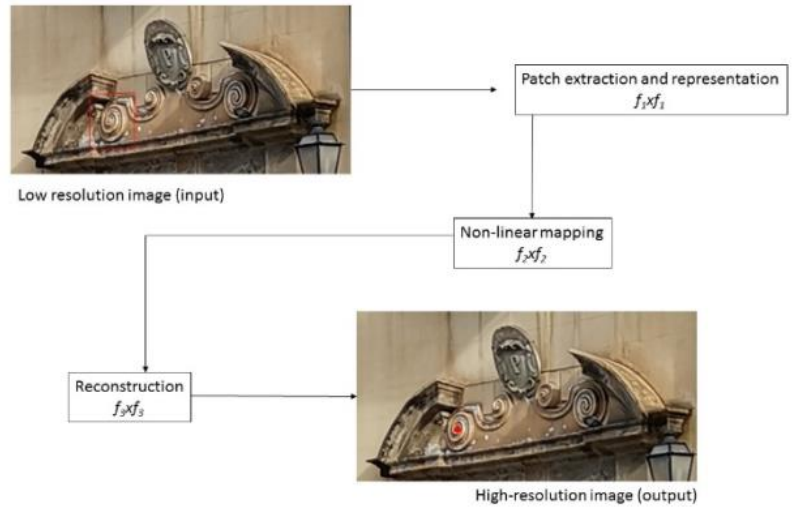

Figure 5: In the picture, there are three different layers: $f_{I} x f_{l}$, $f_{2} x f_{2}, f_{3} x f_{3}$. Starting from a low resolution image (Y), the first functional layer of the process extracts a set of feature maps. The second layer maps these feature maps nonlinearly to highresolution patch representations. The last layer combines the predictions within a spatial neighbourhood to produce the final high-resolution image $\mathrm{F}(\mathrm{Y})$ called Super-Resolution Image.

As you can see (Figure 4,5), this software offers a valid support to achieve the SRI starting from a LI and this is one of the most important feature in image-based modelling (Freedman et al., 2011).

Once we converted all the images from Low- Resolution to Super-Resolution, we can start the image-based reconstruction. As said before, we have to process three data set: the Lowresolution, the High and the Super respectively.

\subsection{SfM reconstruction and comparison}

The data set obtained from the processing of the images, to convert low-resolution images into Super-resolution images, will be used to reconstruct the dense cloud of points of the façade, using Agisoft Metashape Professional software.

The same processing will be applied to the data set with the original high-resolution images. In this way, it will be possible establish the RMS between the dense cloud of both dense clouds generated from data set and verify the reliability of the high resolution processing. In fact, this methodology is widely used in computer vision to the recognition of the images but it was never used to SfM 3D reconstruction.

Moreover, to have a further verification of the validity of the methodology, the dense cloud, using the source data set with the low images, has been elaborated. In addition, this dense cloud will be compared with that obtained from the processing of the high-resolution source data set. In this way, it will be possible to compare the RMS results between the low image origin dense cloud and the high image source one, and the super- resolution image dense cloud, obtained from the processing, and the high resolution source one (Stathopoulou et al., 2019).

In the following figures you can observe the difference between the SfM reconstruction obtained from the different data set, the low and super resolution ones.

The respective dense clouds reconstructed, have been compared to verify the quality of the derived $3 \mathrm{D}$ information, using Cloud Compare v2 (Oniga, et al., 2016). Before upload the dense clouds on Cloud Compare platform, the models have been scaled in Agisoft Metashape during the 3D reconstruction processing (Gonzalez-Aguilera et al., 2020).

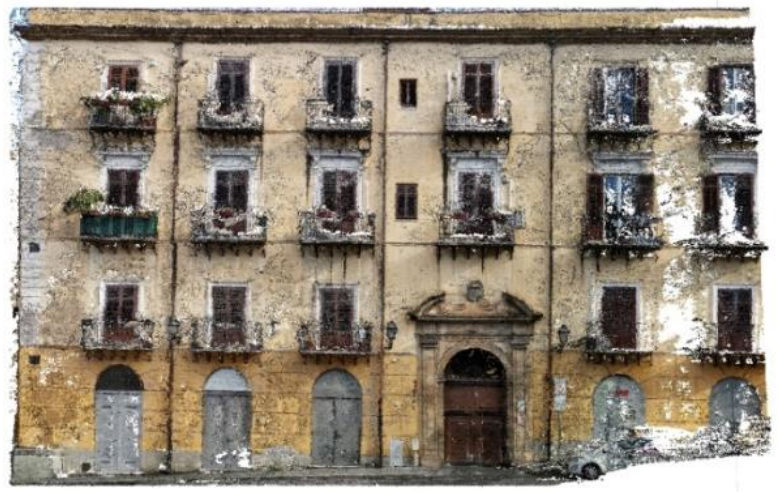

Figure 6: SfM reconstruction using low- resolution image of the façade of the ancient building. Dense Cloud.

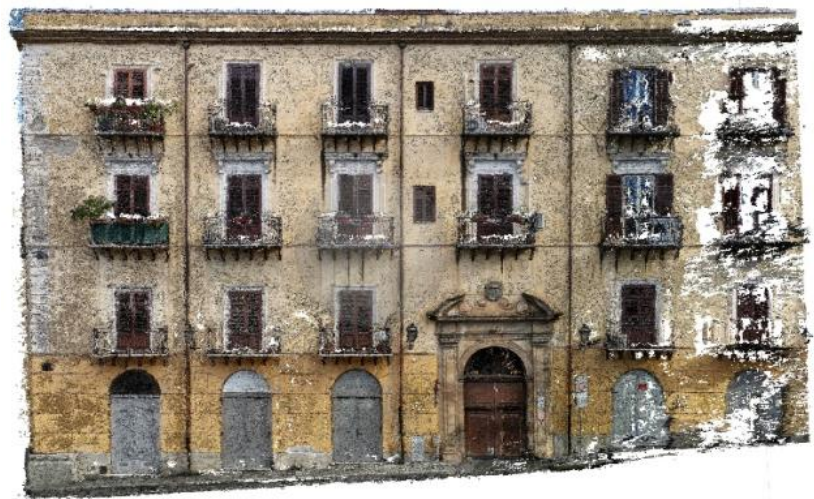

Figure 7: SfM reconstruction using Super- Resolution image of the façade of the ancient building. Dense Cloud.

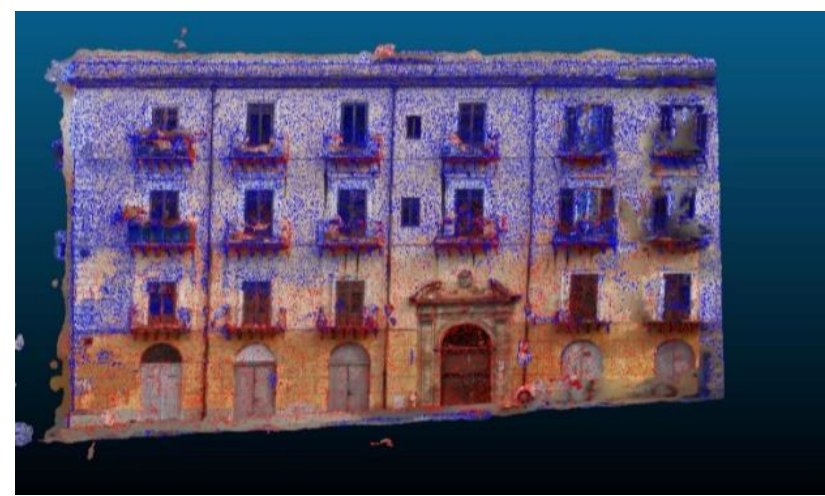

Figure 8: Merge cloud of Low-Image data set dense cloud and ground truth dense cloud. The blue colour indicates a major RMS value than red one.

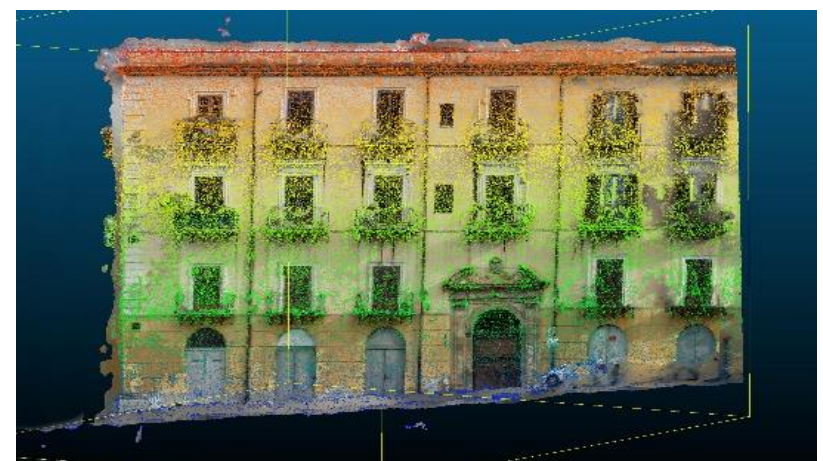

Figure 9: Merge cloud of Super-Image data set dense cloud and ground truth dense cloud. The red colour indicates a major RMS value than blue one 


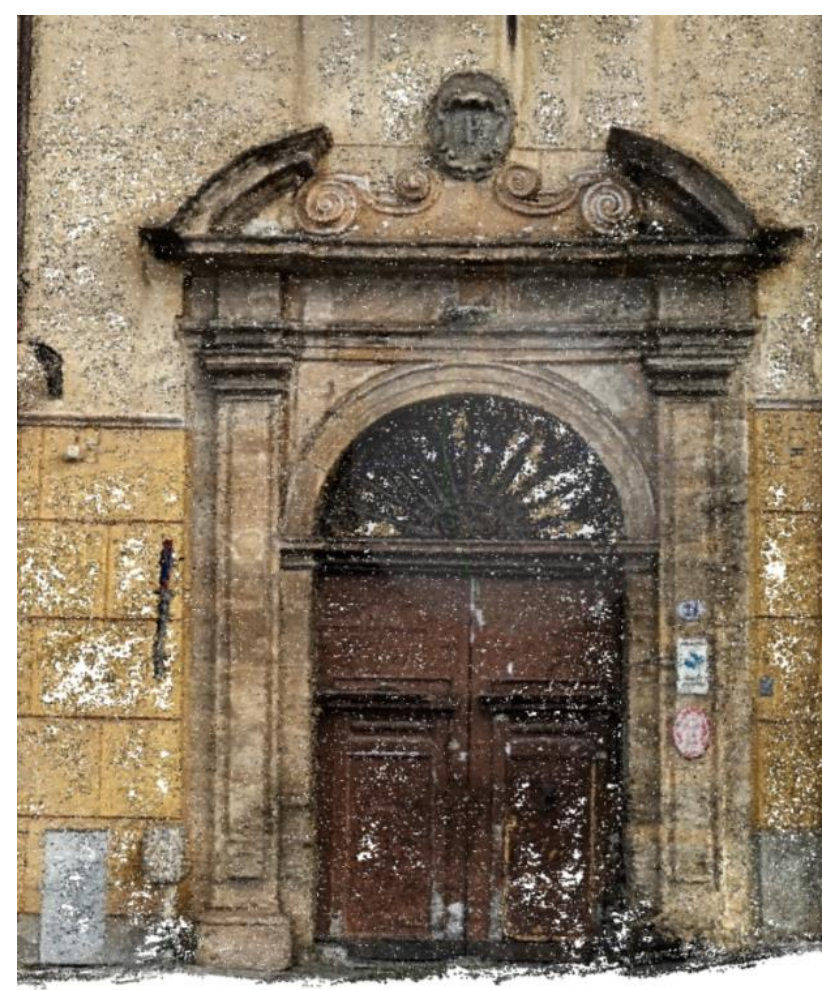

Figure 10: SfM reconstruction using low- resolution image of the portal of the façade of the ancient building. Dense Cloud

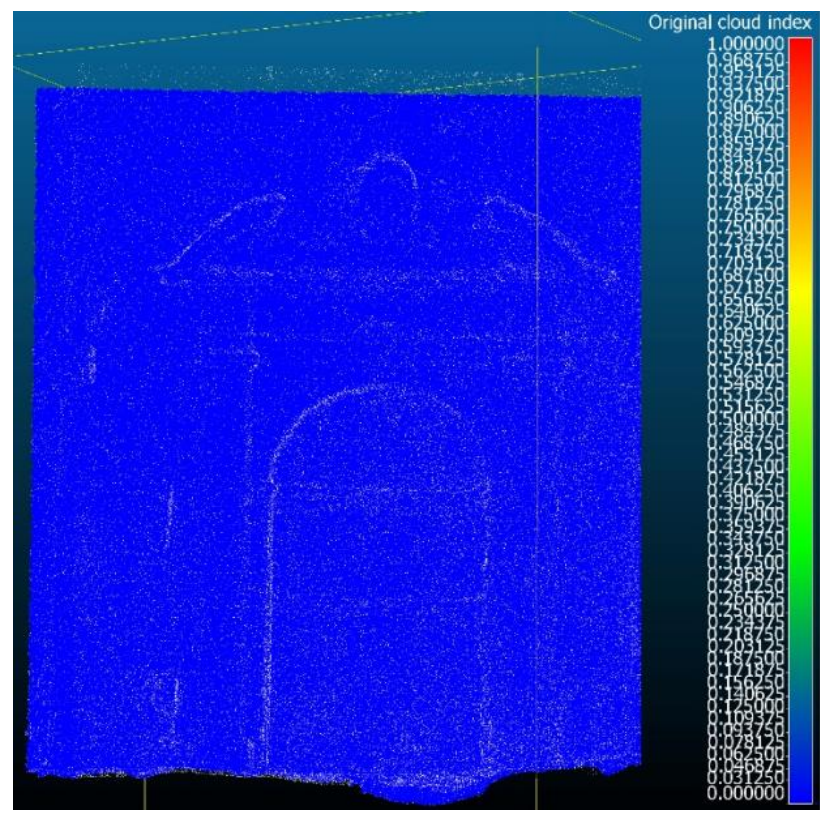

Figure 12: Merge cloud of Low-Image data set dense cloud and ground truth dense cloud. The white colour indicates a major RMS value than blue one.

In addition, the alignment of the different clouds has been conducted using the same (for each different dense cloud) marker points that had been chosen before making the data set (Fukuzono, 1990). Figures 6, 7, 8 and 9 show the dense clouds of the Low and Super resolution data set of the façade (Figures $6,7)$ and merge dense clouds of the respective dense clouds (Figures. 8, 9).

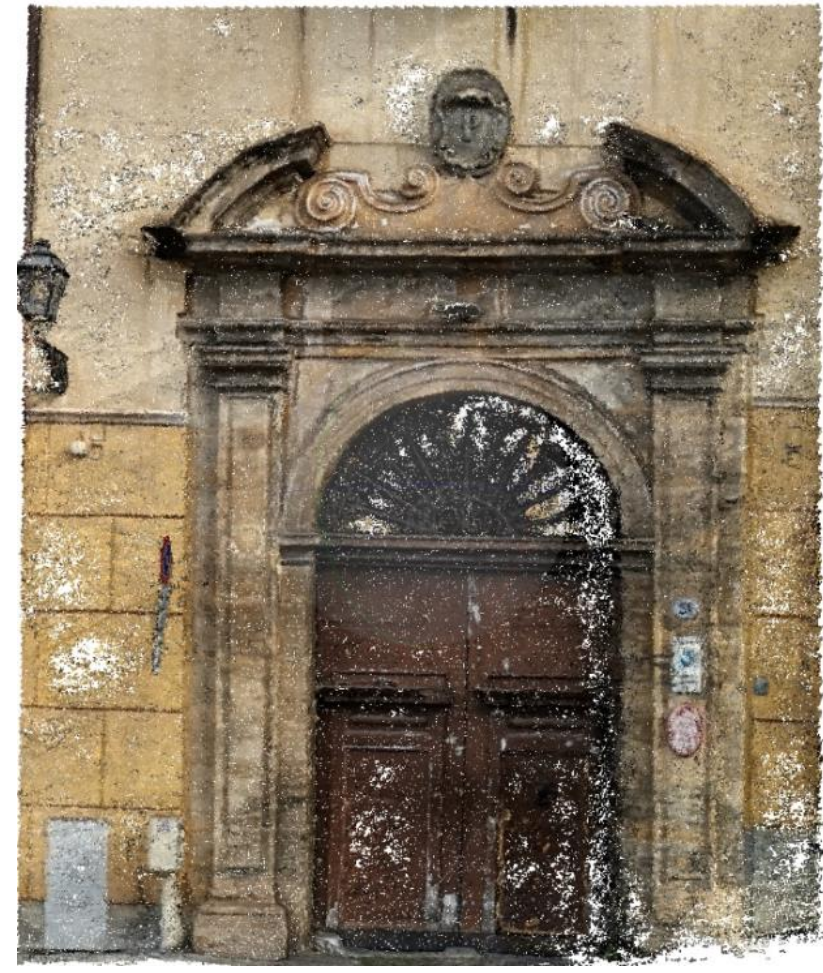

Figure 11: SfM reconstruction using Super- resolution image of the portal of the façade of the ancient building. Dense Cloud.

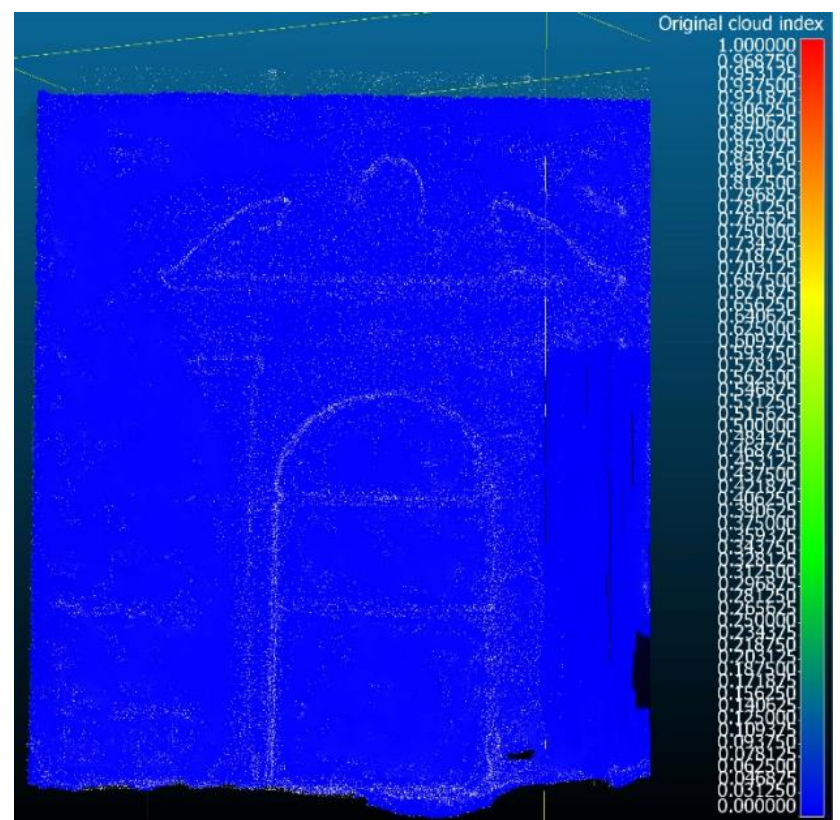

Figure 13: Merge cloud of Super-Image data set dense cloud and ground truth dense cloud. The red colour indicates a major RMS value than blue one.

The merge clouds of the different comparison show that the merge dense cloud obtained from the Super- resolution is closer the truth one, more than the Lower- resolution merge dense cloud.

Figures 10, 11, 12 and 13 show the dense clouds of the Low and Super resolution data set of the portal of the façade (Figures 10 , 11) and merge dense clouds of the respective dense clouds (Figures 12, 13). 
The merge clouds of the different comparison show that the merge dense cloud obtained from the Super- resolution data set, is closer the truth one, more than the Lower- resolution merge dense cloud.

\section{RESULTS}

In this investigation the performance of the Super-resolution image was evaluated, not so much for the recognition of the images, as for the construction of the 3D model from images. The proposed methodology envisaged two main phases: the first one to process the low-resolution images in Super-resolution and the second one to process the data set to 3D reconstruction. Finally, the dense clouds have been compared to verify the quality of the 3D model information. The same picture has been taken from the same point of observation in low and high resolution. The high resolution image was used as ground truth source and the low one was used for the processing in Super- resolution. In other words, the comparison was conduct from the dense cloud generated from the data set processed from low to high and the data set with high resolution source images. The values of RMS achieved, demonstrate that there is a RMS $=0.049$ for the comparison of the entire façade and a RMS $=0.0238154$ for a restricted area as the portal (Tab. 1, Tab 2). This result is predictable if we consider the height in the top part of the building and the difficulty of creating the data set for the urban conditions in front of the building.

\begin{tabular}{|c|c|c|c|}
\hline \multicolumn{2}{|c|}{$\begin{array}{l}\text { Final RMS: } \\
0.0993363\end{array}$} & \multicolumn{2}{|c|}{$\begin{array}{l}\text { Final RMS: } \\
0.0538193\end{array}$} \\
\hline \multicolumn{2}{|c|}{$\begin{array}{l}\text { Transformation } \\
\text { matrix }\end{array}$} & \multicolumn{2}{|c|}{$\begin{array}{l}\text { Transformation } \\
\text { matrix }\end{array}$} \\
\hline 1.562 & 0.379 & 0.613 & -0.143 \\
\hline-0.048 & 20.167 & 0.015 & -14.320 \\
\hline-0.380 & 1.563 & 0.142 & 0.613 \\
\hline-0.025 & -13.025 & 0.019 & 5.011 \\
\hline 0.041 & 0.036 & -0.019 & -0.015 \\
\hline 1.608 & 0.309 & 0.629 & 0.036 \\
\hline 0.000 & 0.000 & 0.000 & 0.000 \\
\hline 0.000 & 1.000 & 0.000 & 1.000 \\
\hline
\end{tabular}

Table 1: RMS [m] value carry out comparing Low- Resolution images data set dense Cloud with the Ground Truth Source one, for façade (on the left side) and the portal (right side).

In fact, for the portal data set the value is significantly reduced. Considering the dimension of the object to survey the result is satisfactory. Furthermore, the result of RMS values for the low resolution data set, demonstrate that applying the SuperResolution algorithm, the RMS values decrease significantly. In other words, in case of low resolution data set, the SuperResolution Image processing, improve the quality of the 3D model (Figures 14, 15).

\begin{tabular}{|c|c|c|c|}
\hline \multicolumn{2}{|c|}{$\begin{array}{l}\text { Final RMS: } \\
0.0494363\end{array}$} & \multicolumn{2}{|c|}{$\begin{array}{l}\text { Final RMS: } \\
0.0238154\end{array}$} \\
\hline \multicolumn{2}{|c|}{$\begin{array}{l}\text { Transformation } \\
\text { matrix }\end{array}$} & \multicolumn{2}{|c|}{$\begin{array}{l}\text { Transformation } \\
\text { matrix }\end{array}$} \\
\hline 1.562 & 0.379 & 0.613 & -0.143 \\
\hline-0.048 & 20.167 & 0.015 & -14.320 \\
\hline-0.380 & 1.563 & 0.142 & 0.613 \\
\hline-0.025 & -13.025 & 0.019 & 5.011 \\
\hline 0.041 & 0.036 & -0.019 & -0.015 \\
\hline 1.608 & 0.309 & 0.629 & 0.036 \\
\hline 0.000 & 0.000 & 0.000 & 0.000 \\
\hline 0.000 & 1.000 & 0.000 & 1.000 \\
\hline
\end{tabular}

Table 2: RMS [m] value carry out comparing Super- Resolution images data set dense Cloud with the Ground Truth Source one, for façade (on the left side) and the portal (right side).

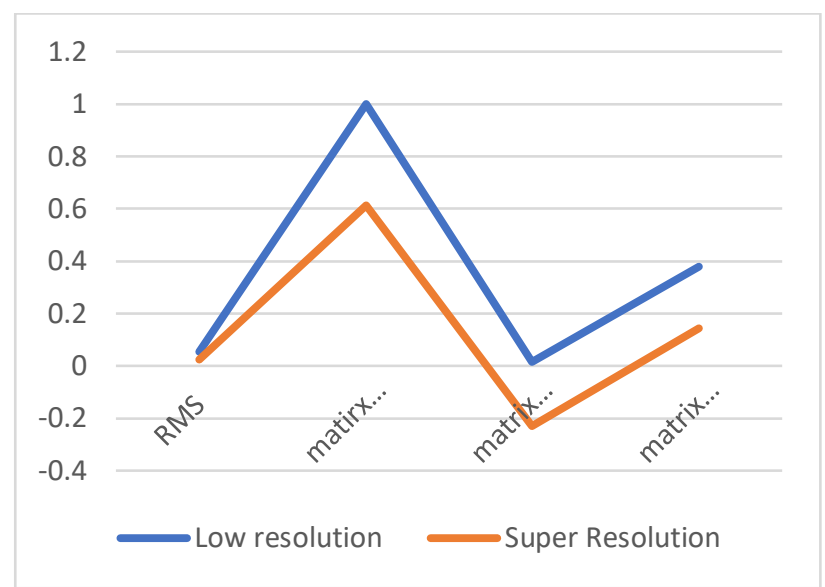

Figure 14: RMS [m] and matrix transformation both low and super resolution images for portal.

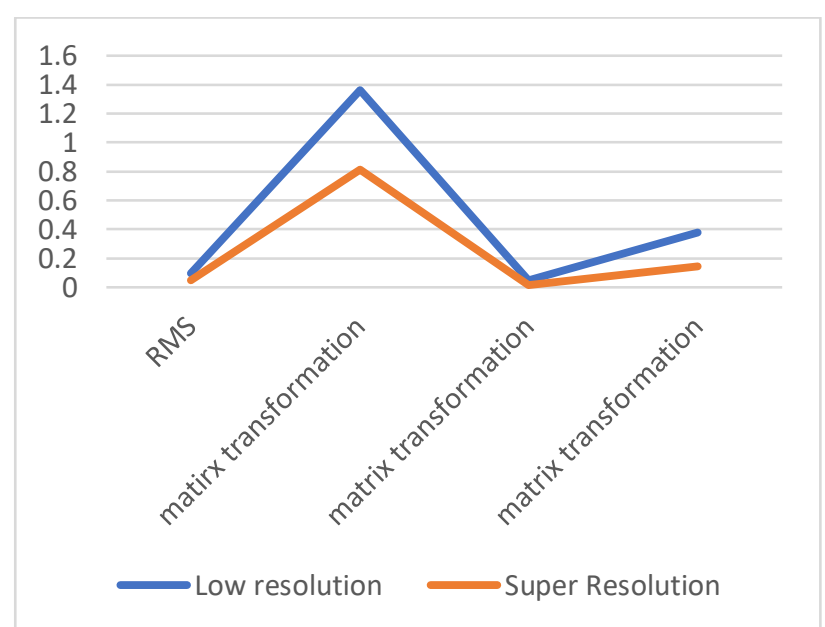

Figure 15: RMS [m] and matrix transformation both low and super resolution images for façade. 


\section{CONCLUSIONS AND FUTURE WORKS}

The results achieved demonstrated that, in case of low-resolution images, is opportune to increase the quality of the source image using the presented Super-Resolution approach to achieve a better quality of the 3D photogrammetric model. The reported RMS values indicated also important improvements thanks to the Super-Resolution approach. Nevertheless, for future improvements, it could be necessary to automate the SuperResolution image generation for big data set to avoid the processing of each one image. The automation of the SuperResolution image creation could increase the diffusion of the methodology.

\section{REFERENCES}

Barazzetti L., 2011. Planar metric rectification via parallelograms In: Proc. Int. Conf. 'Videometrics, Range Imaging, and Applications XI,' 23-26 May, Munich, Germany, Proc. of SPIE, Vol. 8085, No. 80850R, 12 pp. (e-doc).

Fan, B., Kong, Q., Wang, X., Wang, Z., Xiang, S., Pan, C., Fua, P. 2019. A performance evaluation of local features for imagebased 3D reconstruction. IEEE Transactions on Image Processing, 28(10), 4774-4789.

Freedman, G., Fattal, R. (2011) Image and video upscaling from local self-examples. ACM Transactions on Graphics 30(2), 12 Glasner, D., Bagon, S., Irani, M. (2009) Super-resolution from a single image. In: IEEE International Conference on Computer Vision. pp. 349-356

Fan, B., Kong, Q., Wang, X., Wang, Z., Xiang, S., Pan, C., Fua, P. 2019. A performance evaluation of local features for imagebased 3D reconstruction. IEEE Transactions on Image Processing, 28(10), 4774-4789.

Fukuzono, T., 1990. Recent Studies on Time Prediction of Slope Failure. Landslide News, 4: 9-12.

González-Aguilera, D., Ruiz de Oña, E., López-Fernandez, L., Farella, E. M., Stathopoulou, E. K., Toschi, I., Remondino, F., Rodríguez-Gonzálvez, P., Hernández-López, D., Fusiello, A., and Nex, F..2020. Photomatch: an open-source multi-view and multi-modal feature matching tool for photogrammetric applications. ISPRS Int. Arch. Photogramm. Remote Sens. Spatial Inf. Sci., Vol. 43(B5-2020), pp. 213-219. Code available at: https://github.com/TIDOP-USAL/photomatch

Kim, K.I., Kwon, Y. 2010. Single-image super-resolution using sparse regression and natural image prior. IEEE Transactions on Pattern Analysis and Machine Intelligence 32(6), 1127-1133

Jin, Y., Mishkin, D., Mishchuk, A. et al., 2020. Image Matching Across Wide Baselines: From Paper to Practice. Int Journal of Computer Vision, Vol. 129, pp. 517-547

Inzerillo, L. 2020. SfM Techniques Applied in Bad Lighting and Reflection Conditions: The Case of a Museum Artwork Advances in Intelligent Systems and Computing 943, pp. 394401

Lowe, D.G., 2004. Distinctive Image Features from Scale Invariant Keypoints. International Journal of Computer Vision, 60: $91-110$

Luo, Z., Zhou, L., Bai, X., Chen, H., Zhang, J., Yao, Y., Li, S., et al., 2020. ASLFeat: Learning Local Features of Accurate Shape and Localization. Proc. CVPR.
Moulon, P., Monasse, P., Perrot, R. Marlet, R., 2016. OpenMVG: Open multiple view geometry. In International Workshop on Reproducible Research in Pattern Recognition, pp. 60-74.

Qiao, G., Lu, P., Scaioni, M., Xu, S., Tong, X., Feng, T., Wu, H., Chen, W., Tian, Y., Wang, W., and R. Li, 2013. Landslide Investigation with Remote Sensing and Sensor Network: From Susceptibility Mapping and Scaled-down Simulation towards in situ Sensor Network Design. Remote Sensing, 5(9): 4319-4346.

Oniga, E., Savu, A., Negrilă, A., 2016. The evaluation of CloudCompare software in the process of TLS point clouds registration. RevCAD J. Geodesy Cadastre, 21, 117-124.

Ono, Y., Trulls, E., Fua, P., Yi, K.M., 2019. LF-Net: Learning local features from images. Proc. NIPS.

Remondino, F., Nocerino, E., Toschi, I., Menna, F., 2017. A critical review of automated photogrammetric processing of large datasets. ISPRS Int. Arch. Photogramm. Remote Sens. Spatial Inf. Sci., Vol. 42(2/W5), pp. 591-599.

Roberts, R., Inzerillo, L., Di Mino, G. 2020. Using uav based 3d modelling to provide smart monitoring of road pavement conditions. Information (Switzerland). 11(12),568, pp. 1-24

Rublee, E., Rabaud, V., Konolige, K., Bradski, G.R., 2011. ORB: An efficient alternative to SIFT or SURF. Proc. ICCV.

Shen, T., Luo, Z., Zhou, L., Zhang, R., Zhu, S., Fang, T., Quan, L., 2018. Matchable Image Retrieval by Learning from Surface Reconstruction. Proc. ACCV.

Schönberger, J., Frahm, J., 2016. Structure-From-Motion Revisited. Proc. CVPR.

Schönberger, J.L., Hardmeier, H., Sattler, T., Pollefeys, M., 2017. Comparative Evaluation of Hand-Crafted and Learned Local Features. Proc. CVPR.

Schulter, S., Leistner, C., Bischof, H. 2015. Fast and accurate image upscaling with super-resolution forests. In: IEEE Conference on Computer Vision and Pattern Recognition. pp. 3791-3799

Stathopoulou, E.-K., Welponer, M., and Remondino, F., 2019: open-source image-based $3 \mathrm{~d}$ reconstruction pipelines: review, comparison and evaluation. ISPRS Int. Arch. Photogramm. Remote Sens. Spatial Inf. Sci., Vol. 42(2/W17), 331-338.

Verdie, Y., Yi, K. M., Fua, P., Lepetit, V., 2015 TILDE: A Temporally Invariant Learned DEtector. Proc. CVPR.

Timofte, R., De Smet, V., Van Gool, L. (2013) Anchored neighborhood regression for fast example-based superresolution. In: IEEE International Conference on Computer Vision. pp. 1920-1927

Timofte, R., De Smet, V., Van Gool, L. 2014. A+: Adjusted anchored neighborhood regression for fast super-resolution. In: IEEE Asian Conference on Computer Vision

Wu, C., 2013. Towards Linear-Time Incremental Structure from Motion. Proc. 3DV.

Yang, C.Y., Huang, J.B., Yang, M.H. 2010. Exploiting selfsimilarities for single frame super-resolution. In: IEEE Asian Conference on Computer Vision, pp. 497-510

Yang, C.Y., Ma, C., Yang, M.H. 2014. Single-image superresolution: A benchmark. In: European Conference on Computer Vision, pp. 372-386 
Yang, J., Lin, Z., Cohen, S. 2013. Fast image super-resolution based on in-place example regression. In: IEEE Conference on Computer Vision and Pattern Recognition. pp. 1059-1066

Yi, K.M., Trulls, E., Lepetit, V., Fua, P., 2016. LIFT: Learned invariant feature transform. Proc. ECCV. 